\title{
The concept of biomechatronic systems as a means to support the development of biosensors
}

\section{Opinion}

Biomechatronics acts on a broad spectrum of scientific and industrial fields, ranging from biomimetics to biomedical engineering. In these fields mainly but not excluively scientists from biology and medicine interact with engineers, computer scientists and physicists, and it becomes obvious, that the term ,biosensors“ as base of understanding far beyond its IUPAC-definition owns two general perceptions. Bio: something in the context of living systems, from molecule via cells and organisms to environment. Sensor: something peripheral for acquisition of signals, basic for gaining data and after their processing information. Biosensor: sensitive organ in an organism, or something technical, inspired by observation of living nature (biomimetics), or something technical to observe biological phenomena, or something biological, integrated into something technical, to observe something biological or technical. To start common work of life scientist and technicians under such non-uniform definitions and conditions in consent on a scientifically acceptable fundament, we made good experiences refering to the theory of systems, widely accepted in science, and deriving the details and their denotations from it. Our proposal for a system structure, applicable as well to biosystems as to technical systems (and their hybrids) ${ }^{1}$ owns a symmetrical structure what concerns sensors and actors (Figure 1).

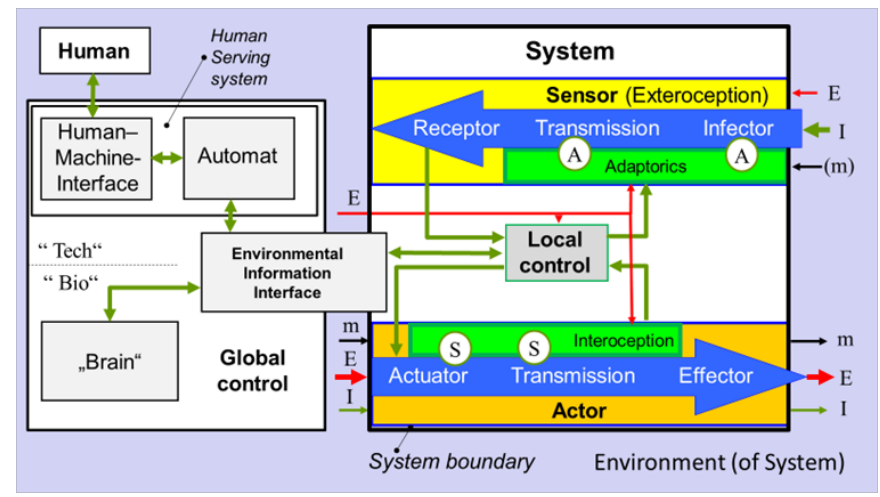

Figure I Biomechatronic system.

The sensor-actor system under study (,System") is defined by the system boundary (thick black line), crossed by flows of energy $E$ (red arrows), mass $m$ (black) and information (green). In the environment of this system, the „Global control“ as a seperate system interacts via an „Environmental Information Interface“. The global control is abstracted for a biological solution (,Bio“: „Brain“) and a technical solution (,Tech": an „Automat“). This automat may interact in a „Human Serving System“ with human beings via a „HumanMachine-Interface“. The sensor chain (yellow) is actuated (,A“, „Adaptorics“, light green), the actor chain (orange) is sensorized (,S“, ,Interoception“, light green).

The system under study owns actors, sensors and local control (substrate e.g. biologically spinal cord, technically $\mu \mathrm{C}$ ). System boundaries are crossed by flows of mass (m), energy (E) and information (I). The local control interacts with the global control (e.g. biologically brain, technically PC), which is part of the system's environment (or may be integrated into the system, if senseful for
Volume 2 Issue 4 - 2017

\author{
Hartmut Witte, Cornelius Schilling \\ Institut für Mechatronische Systemintegration, Germany
}

Correspondence: Hartmut Witte, Fachgebiet Biomechatronik, Institut für Mechatronische Systemintegration, Fakultät für Maschinenbau,Technische Universität IImenau, Germany, Email Hartmut.Witte@tu-ilmenau.de

Received: April 27, 2017 | Published: April 28, 2017

the processes to be analysed or synthesized) via an Environmental Information Interface (EII). What is different from the standard models, is the basic supposition of actuation of sensors, and sensorization of actors.

The „sensor“ in common sense (biologically the eye, technically the component measuring accelerations) is separated into three main components. The ,infector" (the term mirrors the well established term „effector" on the actor side; latin ,inferre“ means "to carry to the inside“, „effere“ „to carry to the outside“) is the sensory interface to the environment, forming a path for information (primary signal) to enter the body: e.g. the Cornea allows light to enter the eye. The transmission guides and transforms (quality, quantity, even modality) the signal on the spatial and temporal way to the sensor sensu strictu, the „receptor": to keep the example, anterior ocular chamber, iris diaphragm, ocular lens, posterior ocular chamber, vitreus body guide the signal to the retina. Within the transmission, no amplification (no positive change of energy content of the signal) occurs. Both infector and transmission form, ,adaptorics ${ }^{\text {(2) }}$ ) the structures to adapt the primary signal to the needs of the sensor, which is capable to process a limited range of properties of a (in our chain secondary) signal. Thus in animals a wide variety of mechanical signals may be detected by only a small number of main sensor types (which at last are nervous endings covered by standard type of the parts of the transmission near to the nerve, e.g. Vater-Pacini bodies). This complete sensor chain in many technical sensor systems may be identified (e.g. in a camera as an equivalent resp. biomimetic copy of a human eye), but must not necessarily.

The concept of the actoric chain as well is inspired by analyses of bioactoric systems like muscles, but modern mechatronic systems contain the elements as well - more an effect of functional and structural convergence using the same physics than of bio-inspiration. Since internationally the terms ,actor", ,actorics“ or ,actuator" are used in any imaginable hierarchical order, we choose their application in a way keeping the symmetry to the terminoly in the sensor chain. Thus the "actor" is composed of three components as well: the „actuator“, again a „transmission“, and an „effector" as the contact element to the system's environment. The actuator changes the energy content of the actor chain, inducing energy from energy buffers (e.g. biologically ATP, technically accumulators) into the system: drives, motors, respectively dissipating energy: brakes, dampers. In the biological paragon „muscle“ both functions are carried by 
one structure. In automotive products, the integration of starter and dynamo is a comparable example. The transmission transfers energy, and perhaps modifies its manifestation: in a diesel-electric locomotive the Diesel engine is the actuator, the dynamo and the electric motors are parts of the transmission. In an animal's locomotor apparatus, the muscles change the energy content, but as well serve as parts of the transmission (compliant structures in- and outside the sarcomeres as the actuators sensu strictu, like titin and collagen), and tendon-jointbone complexes are gears of type one of mechanical machines (lever gears; type two makes use of wedge gears) - „exchange“ elements for the power components torque and angular velocity. The energy is transferred into the environment by effectors.

Like the sensor chain is actuated, the actor chain is sensorized (e,g. by muscle spindles, sensors for rotational speed on shafts). Breaking the symmetry of the system model, in the actor chain the sensor for actuator and transmission are merged to one function: interoception. The observance of the effector (part of exteroception) is realized by a sensor chain (often, but not necessarily the one within the system: real systems often are more complex and thus show meshed structures).

The local control gains signals from the sensor chain and the interoception, and steers the actor chain and the adaptorics. It embodies the information interchange with the global control via an „environmental interface“. In the central nervous system, where the spinal cord is part of this system construct and the brain is not, for the sensory information flow here the Thalamus has to be taken into account, for the actoric one Cerebellum and basal ganglia. This example decovers the limitedness of such a simple model construct - a model always is a mapping to reduce complexity of the reality, here: the object under study. The degree of reduction is depending of the task the model has to fulfill, our simple model should serve to pave the way of interdisciplinary exchange, not to explain substrates of natural or artificial intelligence.

In how far this system concept is helpful in the context of biosensors? Biosensors realized in biotechnology as in microsystem technology: the binding structurs define the sensitivity and specifity of the sensor - the infector component of the sensor chain. Biomedical engineering: in the artificial Pancreas, the unsolved problem is the closing of the „window" into the body by immune defense - the infector is impaired. Biomimetics: artificial whiskers intensely gain quality, if the principles of compliance and surface structurization in the paragon are transferred correctly - optimization of the infector. For high-grade integration of sensor systems, high potential is provided by optimization of adaptorics, combined with standard receptors.

\section{Acknowledgements}

None.

\section{Conflict of interest}

The author declares no conflict of interest.

\section{References}

1. De La Mettrie, Julien Offray. L'Homme Machine. Netherlands: Springer; $1747 \mathrm{p}$.

2. Janocha, Hartmut. Adaptronics and Smart Structures. Germany: Springer-Verlag Berlin Heidelberg; 2007. p. 1-535. 